\title{
Chapter 19 \\ Monte Carlo Evaluation of Internal Dose and Distribution Imaging Due to Insoluble Radioactive Cs-Bearing Particles of Water Deposited Inside Lungs via Pulmonary Inhalation Using PHITS Code Combined with Voxel Phantom Data
}

\author{
Minoru Sakama, Shinsaku Takeda, Erika Matsumoto, Tomoki Harukuni, \\ Hitoshi Ikushima, Yukihiko Satou, and Keisuke Sueki
}

\begin{abstract}
The role of this study in terms of health physics and radiation protection has been implemented to evaluate the internal dose (relative to the committed equivalent dose) and the dose distribution imaging due to gamma rays (photons) and beta particles emitted from the radioactive Cs-bearing particles in atmospheric aerosol dusts deposited in the lungs via pulmonary inhalation. The PHITS code combined with voxel phantom data (DICOM formats) of human lungs was used. We have dealt with the insoluble radioactive Cs-bearing particles of water (about $\phi 2.6 \mu \mathrm{m}$ diameter) migrated onto any of six regions, ET1, ET2, BB, AI-bb, LNET, and LNTH, in a respiratory system until dropping into blood vessels. Source parameters were those of an adult male breathing a typical air volume outdoors; in the simulated atmosphere (such as systematically setting up a field) those particles would be released on 21:10 March 14 to 9:10 March 15, 2011 in Tukuba, Japan,
\end{abstract}

\footnotetext{
M. Sakama $(\bowtie)$

Department of Radiation Science and Technology, Institute of Biomedical Sciences, Tokushima University Graduate School, Kuramoto-cho 3-18-15, Tokushima, Japan e-mail: minorusakama@tokushima-u.ac.jp

S. Takeda • E. Matsumoto

Department of Radiation Science and Technology, Institute of Biomedical Sciences, Tokushima University Graduate School, Tokushima, Japan

T. Harukuni • H. Ikushima

Department of Therapeutic Radiology, Institute of Biomedical Sciences, Tokushima University Graduate School, Tokushima, Japan
}

Y. Satou • K. Sueki

Faculty of Pure and Applied Science, University of Tsukuba, Tsukuba, Japan 
as a filter sampling condition already reported by Adachi et al. In this chapter, we discuss the internal dose and the dose distribution imaging in each voxel phantom for human lung tissues corresponding to the respiratory tracts of BB and AI-bb, respectively.

Keywords Monte Carlo simulation • Committed equivalent dose • Cesium-134 - Cesium-137

\subsection{Introduction}

At 14:46 JST (Japan Standard Time) March 11th, 2011, a huge earthquake (magnitude $M \mathrm{~s}=9.0$ at the epicenter; epicenter depth is about $10 \mathrm{~km}$ ) occurred at sanriku off the coast of eastern Japan. A Great tsunami followed the earthquake and caused serious accidents at the Fukushima Daiichi Nuclear Power Plant (FDNPP) and plant workers had been unable to control the cooling system of nuclear fuels at all the plants 1,2,3, and 4. At that time, great amounts of hydrogen gases had been produced around their nuclear fuel rods and the remaining fuel assemblies had not cooled off, filling the plants with hydrogen gases; after a while the emergency condition caused hydrogen explosions at plants 1 (on March 12, 2011) and 3 (on March 14, 2011), and fires also occurred at plants 2 and 4 due to those explosions. These serious accidents had caused the release of large amounts of radioactive materials into the environment together with plumes.

A few years after this accident, we had slightly understood some findings regarding all the radioactivity for released radioactive cesium isotopes of Cs-134 and Cs-137 into the environment and the exact migrations for the radioactive plumes including those radioactive materials upon atmospheric conditions [1-5]. Four years elapsed and it has now become clear that the radioactive materials have chemical and physical properties concerning chemical forms, particle sizes, shape, phases (gas or aerosol), water solubility, and residence time [6-10]. Adachi et al. [11] reported that they directly observed spherical Cs-bearing particles emitted during a relatively early stage (March 14-15) of the accident, and also stated that the spherical Csbearing particles were larger (their diameters were approximately $2 \mu \mathrm{m}$ ), and they were less water soluble than sulfate particles. In their report, they investigated the coexistence of spherical Cs-bearing particles with $\mathrm{Fe}, \mathrm{Zn}$, and possible other elements using SEM and EDS mapping images with the elemental analysis spectrum.

In addition, Satou et al. [12] similarly reported that they investigated whether specific particles such as the spherical Cs-bearing particles observed by Adachi et al. were included in soil samples at the northwestern area about $20 \mathrm{~km}$ away from FDNPP where radioactive plumes migrated on March 15, 2011. It was found that their soil samples contained the same spherical Cs-bearing particles indicated by Adachi et al. [11] and their observed particle size and contained elements were about $2 \mu \mathrm{m}$ diameter with Fe, Zn, and Rb using the same SEM and EDS analyses; they concluded that their obtained properties of these particles in the soil samples were consistent with those reported by Adachi et al. [11] 
As some experimental uptakes allow us to understand step by step the chemical and physical properties of the released radioactive Cs-suspending materials taking forms such as spherical Cs-bearing particles into the plumes, we consider that there exists a need to estimate the health effects on the large crowd of people who were outside around FDNPP or away from there when the plumes migrated after the accident. In the course of this research regarding health physics, we have offered some evaluation materials for the health effects based on calculation of the internal dose and the dose distribution imaging due to beta particles and photons emitted from the Cs-bearing particles deposited inside the lungs in pulmonary inhalation using the PHITS ver. 2.76 (Particle and Heavy Ion Transport code System) code [13] combined with voxel phantom data [14] (formed by the DICOM format) of a human lung next to human respiratory tracts.

In the present work, taking source parameters on the calculation includes the radioactive Cs-bearing particles (about $\phi 2.6 \mu \mathrm{m}$ diameter) distributed onto each of six regions, ET1, ET2, BB, AI-bb, LNET, and LNTH, in a human respiratory tract group until dropping into blood vessels on the assumption of one adult male breathing a typical air volume outdoors during March 14, 21:10 (local time) to March 15, 09:10, 2011 in Tukuba, Japan [11]. We have evaluated the internal exposure and the dose distribution imaging in each of the lung voxel phantoms corresponding to the regions of BB and AI-bb, respectively, using the PHITS code. The period of our interest includes an aerosol sampling filter with a maximum radioactivity level of about $1.00 \mathrm{E}+04 \mathrm{mBq} / \mathrm{m}^{3}$ from the radioactive plume and also the radioactive Cs-bearing spherical particles found in the filter, as reported by Adachi et al. [11].

\subsection{Method and Materials}

Considering internal exposure results from intake of an unsealed radioactive material, in this case treated as the radioactive Cs-bearing particles of Cs-134 and Cs-137, to the human body, it seems natural that there are three pathways into the body for the radioactive material to take:

1. Inhalation: entry through organs in a respiratory tract

2. Ingestion: entry through gastrointestinal tract

3. Direct absorption: absorption through intact or wound

It should be noted that the inhalation pathway is most closely connected with a suspending material such as an aerosol cluster in the atmosphere. In the human body, the respiratory tract is mainly in charge of inhalation. To estimate internal exposure at each of the organs or tissues using all calculation methods such as Monte Carlo simulation codes, there is a need for appropriate mathematical models to describe the various processes involved in the internal deposition and retention of the cesium nuclides and the associated radiation doses of gamma rays (photons) and beta particles emitted from these decays received by various organs and tissues 
in the respiratory tract. As a general standard model, the respiratory tract is viewed as a series of compartments into which the cesium-bearing particles enter and exit at various rates, ultimately being removed from the respiratory tract regions through exhalation and mechanical clearance processes to outside the body, gastrointestinal tract, and blood, respectively. We have stated here that a deposited dose at each organ or tissue, which is due to various radiation, should really be equivalent to a "committed equivalent dose" including the well-known one of radiation safety terms. That is, in this study, the committed equivalent dose should be estimated extremely at each compartment composed of respiratory tract regions using the PHITS code based on a Monte Carlo calculation method.

Following the recommendations by the ICRP in publication 60 [15] and the model of the human respiratory tract in publication 66 [16], we had properly specified PHITS calculation parameters in input files on which geometrical definitions of PHITS's own code (so-called PHITS language) were interpreted as compartment models of the human respiratory tracts. This compartment modeling was conformed for children aged 3 months, 1, 5, and 10 years and for male and female 15-year-olds and adults of a general population stated on the ICRP publications. The respiratory tract regions were represented by five regions: the Extrathoracic (ET) airways were divided into ET1, the anterior nasal passage, and ET2, consisting of the posterior nasal and oral passages, the pharynx and larynx. The thoracic regions were Bronchial (BB), Bronchiolar (bb), and AlveolarInterstitial (AI, the gas exchange region). For the bb and AI, inasmuch as both are located next to each other around respiratory terminal bronchioles, in this study we regarded them as a unity region of AI+bb. Lymphatics were associated with the extrathoracic and thoracic airways (LNET and LNTH). Their regions were defined based principally on radiobiological considerations, but also taking account of differences in respiratory function, deposition, and clearance for the generation member. Each PHITS calculation at those six regional compartments, ET1, ET2, BB, AI-bb, LNET, and LNTH, was implemented by 100,000 histories of photon (gamma ray) and beta particle $\left(e^{-}\right)$decaying Cs-134 and Cs-137 nuclides, respectively, with three different types, type-F (fast), type-M (middle), and type-S (slow), of cesium transfer coefficients into a respiratory tract in a body, assuming that the radioactive Cs-bearing particles $(2.6 \mu \mathrm{m}$ diameter $)$ were deposited at each tissue of those six tissue region compartments. The transfer coefficients were obtained by the WinAct package software [17] based on the ICRP publication 66. This package software is the ORNL numerical solver (Windows version) for the coupled set of differential equations describing the kinetics of a radionuclide in the body.

With the present PHITS calculation method of the committed equivalent doses due to the Cs-bearing particles on the respiratory tract regions, it is very important to evaluate $\mathrm{U}(50)$ and SEE (Specific Effective Energy) values. U(50) gives the total number of decaying nuclides which depends on the decay property and the elemental transfer coefficient into a tissue of interest and the value has been 
evaluated using the WinAct software. Setting three types of differences in transfer coefficients from type-F to type-M and type-S, we have estimated the committed equivalent doses upon the three cesium transfer conditions on each type of U(50). SEE values have the relationship among radiation branching ratios, $Y$, radiation energies for gamma rays and average beta particles of Cs-134 and Cs-137, E, specific absorbed fraction, $\mathrm{SAF}$, and radiation weighting factor, $w$, and this value is given in the function:

$$
\mathrm{SEE}=Y \times E \times \mathrm{SAF} \times w .
$$

The general standard method includes the general committed equivalent dose that thus far has been estimated using the SEE value based on the empirically recommended SAF value of ICRP publication 66. Using the present PHITS calculation method, its code has provided the committed equivalent dose on which the PHITS output data directly result in a relative specific absorbed dose per a radiation source event (Gy/source). Therefore, as it is strongly confirmed that it should correspond to the fraction of SAF as shown in Eq. (19.1), the SEE value can be given as involving the SAF value, and we are consequently able to provide the committed equivalent doses ( $\mathrm{Sv} /$ particles) for each respiratory tract using the SEE involved on the calculated SAF value, $Y$, and $w$ in Eq. (19.1).

This PHITS code simulates initial beta and gamma decay properties [18] emitted from Cs-134 and Cs-137 nuclides into the Cs-bearing particles and secondary radiation associated their radiation event by event in a virtualized space. The input files include voxel phantom data compiled for PHITS code into which it has given geometrical and material information of the respiratory tracts and source information of the initial radiations generated uniformly at each respiratory tract of interest as a source region. In other words, the committed equivalent dose has also been provided with the summation of absorbed doses on a lung tissue event by event into a compartment respiratory tract model by adding $\mathrm{U}(50)$ and radioactivity ratio (a ratio of about one to one; this is experimentally well-known from many reports of investigations of specific radioactivity for various environmental samples) of Cs-134 and Cs-137 at an early stage of this nuclear accident to the PHITS input data.

For the present treatment of voxel computational phantoms referred to the adult reference male of ICRP 110 publication [19] we have compiled its CT values [20] formed by the DICOM format to the PHITS input data using the "dicom2phits" package program combined with the PHITS calculation code. This program has involved the data exchange functions based on the relationship between $\mathrm{CT}$ values $[19,20]$ and material densities and compositions at each tissue or organ [14]. In the present PHITS input file, we have generated the voxel phantom data with the smallest unit size of $0.98 \times 0.98 \times 10 \mathrm{~mm}^{3}$ for each compartment of the respiratory tract regions around a human lung. 


\subsection{Results}

\subsubsection{U(50): Total Number of Decaying Nuclides at Respiratory Tracts of $B B$ and $A I$}

Table 19.1 summarized the total numbers U(50) of decaying Cs-134 and Cs-137 nuclides at respiratory tracts of $\mathrm{BB}$ and $\mathrm{AI}$ in comparison with their three different types, type-F (fast), type-M (middle), and type-S (slow). Both the BB and AI tissues shown in Table 19.1, indicate that their U(50) values of type-S (small) for Cs-134 and Cs-137 should become obviously highest with a decreasing cesium transfer coefficient so that the cesium elements have been deposited at each respiratory tract for a long time. In particular, there is a very remarkable difference among the type-F, type-M, and type-S at the AI respiratory tract due to the very large surface area of small alveoli around respiratory bronchioles.

\subsubsection{Internal Exposure Dose Distribution Imaging for a Respiratory Tract of BB}

Figure 19.1a shows the whole systematic upper half of the human body constructed from the voxel phantom data compiled by the PHITS language. The geometrical and material voxel phantom data around a lung are described based on phantom data of an adult male with CT value using the "dicom2phits" package program combined with the PHITS calculation code. The other figures illustrate the calculated internal exposure dose distributions (expressed at the vertical axis of the absorbed dose (Gy) at T-heat mode on PHITS) of type-S in Fig. 19.1b and type-F in Fig. 19.1c for the BB respiratory tract of the present interest. Their absorbed doses expressed into the figures contribute to whole radiation energies absorbed at each tissue due to the primary gamma rays emitted from Cs-134 and Cs-137 decaying nuclides at the BB respiratory tract. The figures represent that both distributed intensities of absorbed dose for type-S and type-F have increased as formed outlines of the compartment boundaries of $\mathrm{BB}$ respiratory tract because of the radiation source generation in there. In addition, in comparison between the figures, it has been found that both generated radiation has been gradually transported on the same order of about $10^{3}$ $10^{4}$ as the intensity distributions across the entire upper half of the body. And

Table 19.1 The total numbers of decaying Cs-134 and Cs-137 nuclides at respiratory tracts of BB and AI

\begin{tabular}{|c|c|c|c|c|c|c|}
\hline \multirow[b]{2}{*}{ Tissue } & \multicolumn{3}{|l|}{ Cs-134 } & \multicolumn{3}{|l|}{ Cs-137 } \\
\hline & Type-F & Type-M & Type-S & Type-F & Type-M & Type-S \\
\hline AI & 275.17 & 2843600 & 10958000 & 271.85 & 3186500 & 23961000 \\
\hline BB & 22.936 & 57487 & 73884 & 22.659 & 58184 & 75069 \\
\hline
\end{tabular}


Fig. 19.1 (a) Shows the entire systematic upper half of the human body constructed from the voxel phantom data compiled by PHITS language. (b, c) Illustrate the calculated internal exposure dose distributions expressed at the vertical axis of absorbed dose (Gy) obtained from T-heat results on PHITS
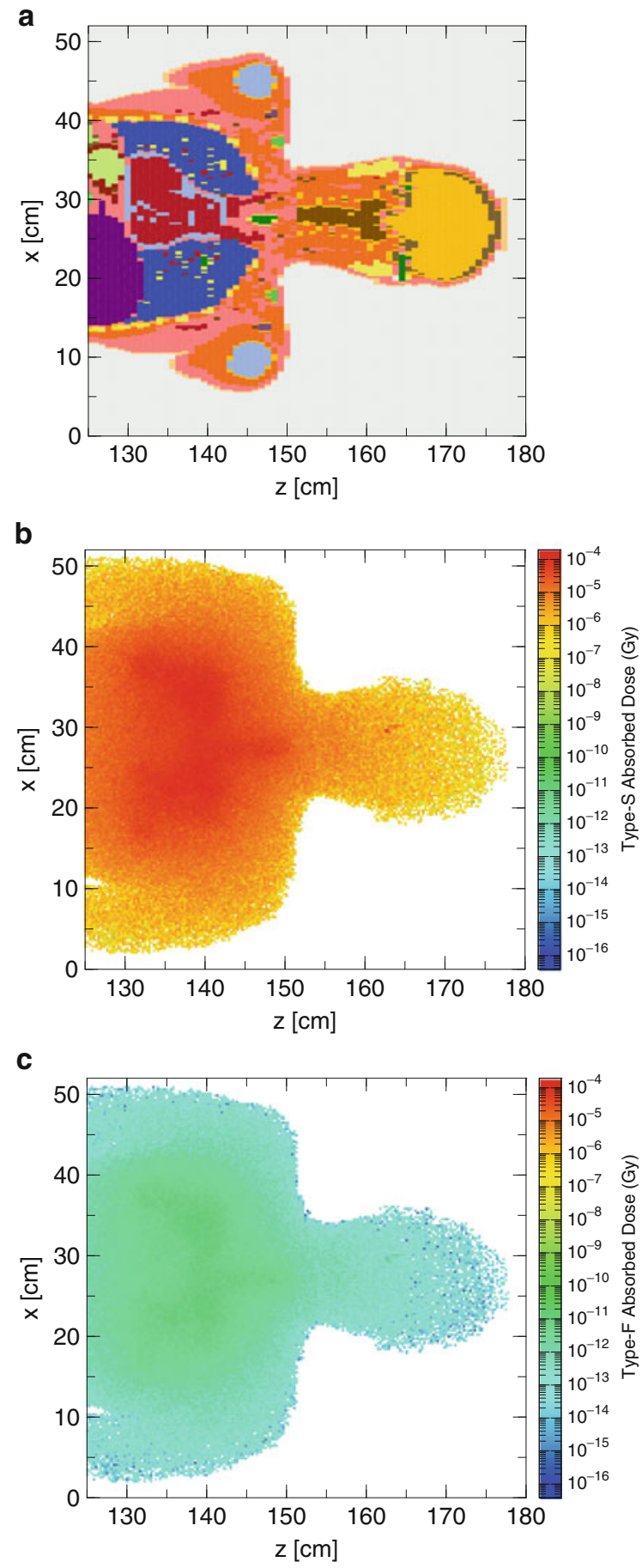
also the absorbed dose distribution of type-S was much higher than that of type$\mathrm{F}$, because it is definitely likely that it was caused by the total number $\mathrm{U}(50)$ of type-S more than that of type-F into the BB region due to too many extremely late Cs-134 and Cs-137 nuclides transferring in the region upon the transfer coefficients of type-S to the blood and gastrointestinal tract.

\subsubsection{Committed Equivalent Doses in Comparison with Dependence on Gamma Rays and Beta Particles Between Cs-134 and Cs-137}

Tables 19.2 and 19.3 show calculated committed equivalent doses in a comparison with dependence on gamma rays and beta particles associated with the decaying nuclides of Cs-134 and Cs-137 between two methods of PHITS and the general standard method, and also among transfer coefficients of type-F, type-M, and type$S$, respectively. In this chapter, we have focused on the calculated result data in a comparison between $\mathrm{BB}$ and $\mathrm{AI}+\mathrm{bb}$ respiratory regions. Comparing all the factors of radiation, transfer coefficient, and respiratory tract in our obtained data as shown in Tables 19.2 and 19.3, it is very likely that the present PHITS simulated method can reproduce those committed equivalent doses estimated approximately by the general standard method. Especially, it is really remarkable that the committed equivalent

Table 19.2 Comparison with calculated committed equivalent doses of Bronchial (BB) and a unity region of Alveolar-Interstitial (AI) and Bronchiolar (bb), which are associated with gamma rays of Cs-134 and Cs-137, by PHITS and general standard method

\begin{tabular}{|c|c|c|c|c|c|c|c|}
\hline & \multicolumn{3}{|c|}{ Cs-134: gamma ray (Sv/particles) } & \multicolumn{3}{|c|}{ Cs-137: gamma ray (Sv/particles) } \\
\hline & & Type-F & Type-M & Type-S & Type-F & Type-M & Type-S \\
\hline PHITS & $\begin{array}{l}\mathrm{BB} \\
\mathrm{AI}+\mathrm{bb}\end{array}$ & $\begin{array}{l}2.85602 \mathrm{E}-11 \\
2.56911 \mathrm{E}-11\end{array}$ & $\begin{array}{l}4.26206 \mathrm{E}-08 \\
3.74353 \mathrm{E}-08\end{array}$ & $\begin{array}{l}1.57344 \mathrm{E}-07 \\
1.41104 \mathrm{E}-07\end{array}$ & $\begin{array}{l}1.60146 \mathrm{E}-11 \\
1.30911 \mathrm{E}-11\end{array}$ & $\begin{array}{l}1.86545 \mathrm{E}-08 \\
1.71161 \mathrm{E}-08\end{array}$ & $\begin{array}{l}1.43411 \mathrm{E}-07 \\
1.29311 \mathrm{E}-07\end{array}$ \\
\hline $\begin{array}{l}\text { General } \\
\text { standard } \\
\text { method }\end{array}$ & $\begin{array}{l}\mathrm{BB} \\
\mathrm{AI}+\mathrm{bb}\end{array}$ & $\begin{array}{l}3.34002 \mathrm{E}-11 \\
2.73125 \mathrm{E}-11\end{array}$ & $\begin{array}{l}3.95500 \mathrm{E}-08 \\
3.76565 \mathrm{E}-08\end{array}$ & $\begin{array}{l}1.45290 \mathrm{E}-07 \\
1.41924 \mathrm{E}-07\end{array}$ & $\begin{array}{l}1.32564 \mathrm{E}-11 \\
1.08211 \mathrm{E}-11\end{array}$ & $\begin{array}{l}1.78343 \mathrm{E}-08 \\
1.70816 \mathrm{E}-08\end{array}$ & $\begin{array}{l}1.36558 \mathrm{E}-07 \\
1.28834 \mathrm{E}-07\end{array}$ \\
\hline
\end{tabular}

Table 19.3 Comparison with calculated committed equivalent doses of Bronchial (BB) and a unity region of Alveolar-Interstitial (AI) and Bronchiolar (bb), associated with beta particles of Cs-134 and Cs-137, by PHITS and general standard method

\begin{tabular}{l|l|l|l|l|l|l|l}
\hline \multicolumn{2}{c}{} & \multicolumn{3}{l}{ Cs-134: $\beta$-particle (Sv/particles) } & \multicolumn{3}{l}{ Cs-137: $\beta$-particle (Sv/particles) } \\
\cline { 3 - 9 } \multicolumn{2}{l|}{} & Type-F & Type-M & Type-S & Type-F & Type-M & Type-S \\
\hline PHITS & BB & $1.62220 \mathrm{E}-11$ & $2.18575 \mathrm{E}-08$ & $4.03264 \mathrm{E}-08$ & $1.71321 \mathrm{E}-11$ & $1.06724 \mathrm{E}-06$ & $7.89293 \mathrm{E}-06$ \\
& AI+bb & $7.15408 \mathrm{E}-12$ & $6.32775 \mathrm{E}-08$ & $2.40142 \mathrm{E}-07$ & $8.40650 \mathrm{E}-12$ & $8.73540 \mathrm{E}-08$ & $6.46041 \mathrm{E}-07$ \\
\hline $\begin{array}{l}\text { General } \\
\text { standard } \\
\text { method }\end{array}$ & BB & $4.21726 \mathrm{E}-10$ & $4.60743 \mathrm{E}-07$ & $6.01231 \mathrm{E}-07$ & $5.86399 \mathrm{E}-10$ & $6.60018 \mathrm{E}-07$ & $9.03500 \mathrm{E}-07$ \\
\hline
\end{tabular}


doses attributed to the gamma rays of Cs-134 and Cs-137 on the PHITS calculation results are entirely consistent with those of the general standard method at both the respiratory tracts of $\mathrm{BB}$ and $\mathrm{AI}+\mathrm{bb}$.

\subsection{Discussion}

In the present study, providing that the radioactive Cs-bearing particles around 21:00 March 14 to 9:00 March 15, 2011, which were probably released from the FDNPP at the early stage of the nuclear accident, are supposed to have been deposited into human lung tissues from the main to tip regions corresponding to $\mathrm{BB}$ and $\mathrm{AI}+\mathrm{bb}$ respiratory tracts, we have discussed that the PHITS method [13] would probably be available for a Monte Carlo evaluation of committed equivalent dose and the relative internal dose distribution imaging based on possible systematic and optional arrangements using the human voxel phantom by itself or a nearby generation, for example, Japanese adult Male (JM) voxel phantom [14], for anyone with health concerns in Fukushima.

Through a course of the present PHITS Monte Carlo simulation method that has calculated the deduced committed equivalent doses for several respiratory tracts using voxel phantom data of adult human lung tissues and the absorbed dose distribution imaging due to gamma rays and beta particles, the Cs-bearing particles, we have confirmed that the calculated committed equivalent doses contributed to the gamma rays of Cs-134 and Cs-137 can fairly reproduce these results led by the general standard method as shown in Table 19.2. From the absorbed dose distribution in Fig. 19.1b, considering that it is very likely that the Cs-bearing particles with less water solubility would belong to the transfer coefficient for Type$\mathrm{S}$, it should be noted that transmitted gamma rays from the BB respiratory tract influence part of the radiation energies on surrounding tissues with a decreasing two to three orders of absorbed dose magnitude of BB itself per voxel into the upper half of the body. In regard to beta particles attributed to Cs-134 and Cs137 in the Cs-bearing particles at both the BB and AI+bb respiratory tracts, as shown in Table 19.3, those committed equivalent doses evaluated by PHITS are inconsistent with those calculated from general standard method for any type, in particular for Type-S, in comparison with the case of the gamma rays. The difference can be explained by the incorrect exchange of all the actual tissue sizes into AI+bb regions to PHITS input data because CT values in the present DICOM data have a minimum-limited unit size for shaping information of these tracts. And thus, the minimum-limited size would be larger than the actual tissue size and it is very likely that there are wide opening spaces between each of the tissues into the AI+bb regions. We have, therefore, deduced that the present PHITS calculation could not reproduce the transport simulations of beta particles for Cs-134 and Cs-137 due to charged beta particles whose property is definitely involved in the stopping power higher than that of non charged gamma rays, and thus the beta particle may be attributed to be greatly influenced by the difference of each opening space size 
around $\mathrm{AI}+\mathrm{bb}$. Then, the rate of transmitting beta particles would increase without these energy losses at AI+bb regions where they should lose the radiation energies themselves.

From the viewpoint of Monte Carlo evaluation of the internal dose and the distribution imaging due to the insoluble radioactive Cs-bearing particles of water deposited in the lung tissues of a human (adult male) via pulmonary inhalation around 21:00 March 14 to 9:00 March 15, 2011, this work will bear out that the PHITS method was able to evaluate that the committed equivalent dose of BB is 1.57344E-07 (Sv/particles) and 4.03264E-08 (Sv/particles) associated with gamma rays and beta particles of Cs-134, 1.43411E-07 (Sv/particles), and 7.89293E-06 (Sv/particles) associated with gamma rays and beta particles of Cs-137, respectively, at Type-S reproducing the migration next to an actual transfer coefficient into lung tissues of the simulated adult male person. Additionally, we have found that the PHITS method is primarily available not only for evaluating the distribution of internal doses of any tissues of interest relative to both the absorbed doses and the committed equivalent doses, but also investigating their doses at any small piece such as a voxel level depending on CT value and taking the gamma ray and beta particle specific spectra to elucidate the dose distribution mechanism in detail; we also have stated that there is a need to improve the time-consuming and statistical precision contributing to more correct calculations using the PHITS code in the future.

\subsection{Conclusion}

We have shown that the present PHITS code, which is combined with voxel phantom data of human lung tissues for an adult male, can approximately evaluate the internal dose relative to the committed equivalent dose and the distribution imaging due to gamma rays and beta particles associated with decaying Cs-134 and Cs-137 at the insoluble radioactive Cs-bearing spherical particles of water probably released during 21:10 March 14 to 9:10 March 15, 2011 in Tukuba, Japan, as reported by Adachi et al. In particular, it was found that the calculated committed equivalent doses due to the gamma-rays of Cs-134 and Cs-137 were entirely consistent with those of the general standard method at both the respiratory tracts of BB and AI+bb using the SEE value based on ICRP publication 66, and the estimated committed equivalent doses were 1.57344E-07 (Sv/particles) and 1.41104E-07 (Sv/particles) for Cs-134 and 1.43411E-07 (Sv/particles) and 1.29311E-07 (Sv/particles) for Cs137 on both Type-S conditions such as a specific transfer coefficient corresponding to the migration rate of cesium until dropping into blood vessels around lung tissues. The PHITS code will probably be available for evaluation of committed equivalent dose and the relative internal dose distribution imaging based on possible systematic and optional arrangements using the human voxel phantom by itself or nearby generation for anyone with health concerns in Fukushima. We plan in the near future to improve several issues of the time-consuming and statistical errors in order to implement more correct calculations by the PHITS method and to extend this method to 
voxel phantom data of the entire human body including various public fields in the environment using the PHITS code coupled with MCAM and RVIS [21].

Acknowledgements The authors are grateful to research group members for radiation transport analysis in Japan Atomic Energy Agency (JAEA), Dr. K. Manabu, Dr. K. Satou, Dr. S. Hashimoto, Dr. T. Furuta, and Dr. T. Satou, for operating voxel phantom data in extremely large file sizes for an adult male and also for implementing the PHITS calculations again and again to make an attempt to optimize the calculation method.

Open Access This chapter is distributed under the terms of the Creative Commons Attribution Noncommercial License, which permits any noncommercial use, distribution, and reproduction in any medium, provided the original author(s) and source are credited.

\section{References}

1. Manolopoulou M, Vagena E, Stoulos S, Ioannidou A, Papastefanou C (2011) Radioiodine and radiocesium in Thessaloniki, Northern Greece due to the Fukushima nuclear accident. J Environ Radioact 102:796-797

2. Amano H, Akiyama M, Chunlei B, Kawamura T, Kishimoto T, Kuroda T, Muroi T, Odaira T, Ohta Y, Takeda K, Watanabe Y, Morimoto T (2012) Radiation measurements in the Chiba Metropolitan area and radiological aspects of fallout from the Fukushima Dai-ichi nuclear power plants accident. J Environ Radioact 111:42-52

3. Momoshima N, Sugihara S, Ichikawa R, Yokoyama H (2012) Atmospheric radionuclides transported to Fukuoka, Japan remote from the Fukushima Dai-ichi nuclear power complex following the nuclear accident. J Environ Radioact 111:28

4. Katata G, Chino M, Kobayashi T, Terada H, Ota M, Nagai H, Kajino M, Draxler R, Hort MC, Malo A, Torii T, Sanada Y (2015) Detailed source term estimation of the atmospheric release for the Fukushima Daiichi nuclear power station accident by coupling simulations of an atmospheric dispersion model with an improved deposition scheme and oceanic dispersion model. Atmos Chem Phys 15:1029-1070

5. Haba H, Kanaya J, Mukai H, Kambara T, Kase M (2012) One-year monitoring of airborne radionuclides in Wako, Japan, after the Fukushima Dai-ichi nuclear power plant accident in 2011. Geochem J 46:271-278

6. Doi T, Masumoto K, Toyoda A, Tanaka A, Shibata Y, Hirose K (2013) Anthropogenic radionuclides in the atmosphere observed at Tsukuba: characteristics of the radionuclides derived from Fukushima. J Environ Radioact 122:55-62

7. Miyamoto Y, Yasuda K, Magara M (2014) Size distribution of radioactive particles collected at Tokai, Japan 6 days after the nuclear accident. J Environ Radioact 132:1-7

8. Kaneyasu N, Ohashi H, Suzuki F, Okuda T, Ikemori F (2012) Sulfate Aerosol as a potential transport medium of radiocesium from the Fukushima nuclear accident. Environ Sci Technol 46:5720-5726

9. Niimura N, Kikuchi K, Tuyen ND, Komatsuzaki M, Motohashi Y (2015) Physical properties, structure, and shape of radioactive Cs from the Fukushima Daiichi nuclear power plant accident derived from soil, bamboo and shiitake mushroom measurements. J Environ Radioact 139:234 239

10. Yanaga M, Oishi A (2015) Decontamination of radioactive cesium in soil. J Radioanal Nucl Chem 303:1301-1304

11. Adachi K, Kajino M, Zaizen Y, Igarashi Y (2013) Emission of spherical cesium-bearing particles from an early stage of the Fukushima nuclear accident. Sci Rep 3:2554:1-5 
12. Satou Y, Sueki K, Sasa K, Kitagawa J, Ikarashi S, Kinoshita N (2015) Vertical distribution and formation analysis of the ${ }^{131} \mathrm{I},{ }^{137} \mathrm{Cs},{ }^{129 m} \mathrm{Te}$, and ${ }^{110 m} \mathrm{Ag}$ from the Fukushima Dai-ichi nuclear power plant in the beach soil. J Radioanal Nucl Chem 303:1197-1200

13. Sato T, Niita K, Matsuda N, Hashimoto S, Iwamoto Y, Noda S, Ogawa T, Iwase H, Nakashima H, Fukahori T, Okumura K, Kai T, Chiba S, Furuta T, Sihver L (2013) Particle and heavy ion transport code system, PHITS, version 2.52. J Nucl Sci Technol 50:913-923

14. Sato K, Noguchi H, Emoto Y, Koga S, Saito K (2007) Japanese adult male voxel phantom constructed on the basis of CT images. Radiat Prot Dosimetry 123(3):337-344

15. International Commission on Radiological Protection (1991) 1990 recommendations of the international commission on radiological protection. Publication 60. Annals of the ICRP, vol 21/1-3. Pergamon Press, Oxford

16. International Commission on Radiological Protection (1994) Human respiratory tract model for radiological protection. Publication 66. Annals of the ICRP, vol 24/1-3. Pergamon Press, Oxford

17. Leggett RW et al (1993) An elementary method for implementing complex biokinetic models. Health Phys 64:260-278

18. Firestone RB, Shirley VS (eds) (1996) Table of isotopes, 8th edn. Wiley, New York

19. International Commission on Radiological Protection (2009) 1990 adult reference computational phantoms. Publication 110. Annals of the ICRP, vol 39/2. Pergamon Press, Oxford

20. Schneider W (2000) Correlation between CT numbers and tissue parameters needed for Monte Carlo simulations of clinical dose distributions. Phys Med Biol 45:459-478

21. Wu Y, FDS Team (2009) CAD-based interface programs for fusion neutron transport simulation. Fusion Eng Des 84:1987-1992 